\title{
Optimal Design of Space Grid Structure
}

\author{
Zi-ying $\mathrm{LI}^{1,}$, , Hong $\mathrm{GAN}^{2, \mathrm{~b}}$ \\ ${ }^{1,2}$ College of Civil Engineering \& Architecture, Anhui Polytechnic University. \\ Wuhu 241000, China \\ aliza1215@yeah.net, ${ }^{\text {b gh@@ahpu.edu.cn }}$
}

Keywords: Optimal design, ANSYS software, Space grid structure.

Abstract. Explained the mathematics process of optimum design of structures in principle .Introduce the concept and course of ANSYS optimum design. Discuss how to use the optimum function of ANSYS and the watchful proceeding. The algorithm theory of first order method in ANSYS optimization method is analyzed, and the effectiveness of ANSYS optimization results is verified by the optimization analysis of a space truss structure, and it also shows that the optimal design can save the material in the condition of satisfying safety.

\section{Introduction}

The optimization design is a new subject in the beginning of 1960s, and it is also a new technology and new design method, which applies the optimal principle and computing technology to the design of the neighborhood, and provides an important scientific design method for engineering design. By using this method, people can find the best design scheme from many design schemes and the efficiency and quality of the design can be improved greatly[1].

There are two ways to optimize the design: the analytic method, which obtain the minimum value by solving the differential and the extreme value; the numerical method, which obtain the minimum value through the iterative approximation with the help of computer and finite element. The analytic method needs to list the equations and solve the differential equations, so it is difficult to solve the complicated problems and often used in theory. According to the different types of design variables, the optimization method has been optimized by the lower level of size, to the higher level of structural shape optimization, which has now reached a higher level of topology optimization. The algorithm is also improved from simple criterion method, to the mathematical programming method, and then to the genetic algorithm.

Now, the long-span structures like gas station roof and airport waiting hall roof structure often adopt the grid structure, the main material of which also use steel structure. There are not many columns in the middle of the grid structure, so it can get a lot of space. All components of grid structure are standardized, which can be directly produced in the factory, so that the component cost is relatively low and the quality is easier to guarantee. Because of these advantages, the grid structure is widely used. Therefore, the optimal design of the grid structure is very practical and necessary.

\section{Basic principle and model of optimal design}

The basic principle of the optimization problem is to establish the optimal model, and then select some optimization method to calculation in the condition of satisfying the design requirements [2]. Finally, the extreme value of the objective function is obtained, and the optimal design scheme is obtained.

There are three main elements in structural optimization design: design variables, state variables and objective functions. The general expression of the mathematical model is:

$$
\begin{array}{ll}
\text { Design variables } & X=\left[x_{1}, x_{2}, \ldots, x_{n}\right]^{T} \\
\text { Objective functions } & f=f(x) \rightarrow \min (\max ) \\
\text { State variables } & h_{j}(x)=0 \quad j=1,2, \cdots, k \\
& g_{i}(x) \leq 0 \quad i=1,2, \cdots, m \quad X \geq 0
\end{array}
$$


Design variables: design variables that need to be continuously adjusted in the design process. Each design variable may have a lower bound, which is used to specify the range of design variables. Common design variables always have the width, the height, and so on.

State variables: the numerical value of the constraint design, which can be a function of the design variables, and can be independent of the design variables. The state variable may have upper and lower limit, and it also may have only a unilateral limit, that is, only the upper or lower limit. Stress can not exceed the allowable stress and deformation can not exceed the specified size and amplitude limit, which usually can be seen as state variables.

Objective function: minimization design of variable parameters, which is should be a function of the design variables, namely, when changing the values of the design variables, the value of the objective function will be changed at the same time.

\section{The principle and procedure of ANSYS optimization design}

(1) Optimization design principle of AYSYS

The basic elements of the optimization design of ANSYS include: design variables, objective function, constraint condition, state variable, design sequence, reasonable and unreasonable design, analysis file, loop, iteration, etc.

The optimization methods provided include: zero order method, first order method and the method of user provided. The zero order method is used to transform the problem into an unconstrained optimization problem by adding penalty function to the objective function, and then to establish the relationship between objective function and design variables by using curve fitting; The First order method is used to transform the problem into a non constrained optimization problem by adding penalty function to the objective function [3]. Then, the partial derivative of the design variable is calculated. The search direction is determined, and the line search method is used to minimize the problem. The search space of the zero order method is large, so it is not easy to get the global optimal solution, and the choice of the starting point has a great impact on the results. The first order method requires the use of the first derivative of the dependent variable; therefore, it is not suitable for the optimization of the objective function of the first order partial derivative. Concrete problems should be analyzed concretely. In this paper, we study the problem by using the first order method to get a better solution.

(2) Optimization design procedure of ANSYS

A typical ANSYSY optimization process usually takes the following steps to complete:

1)Description of engineering problems: the nature of the material and geometric conditions, external force, boundary conditions. 2)Parametric modeling: the data (design variables) that are to be involved in the optimization are defined as the model parameters. 3)Solution: loading and solving the structural parametric model. 4)Post-processing: the state variable values (constraints) and objective function values (optimization objective) were extracted from the optimized processor for parameter evaluation; 5)Optimization parameters evaluation: according to the optimized parameters of this cycle and the optimal parameters of the last cycle, we determine whether the objective function of the loop is optimal. If it is the optimal, the completion of the iteration and exit the optimization cycle to the 7) step, otherwise to the next step; 6)Fixed design variable and re-investment the cycle; 7)Get the optimal design variable values and objective function values.The design flow is shown in Fig. 1:

(3) First order optimization method of ANSYS

The first order optimization method in the optimization process requires the use of the objective function and state variables for the design variables of partial derivatives, so called first order method [4]. First order method can be used to transform the constrained conditions into a penalty function, and the objective function is divided into two parts: the objective function and penalty function.

As shown in Eq. 1:

$$
Q(x, q)=Q(x)+Q(x, q) \text {. }
$$




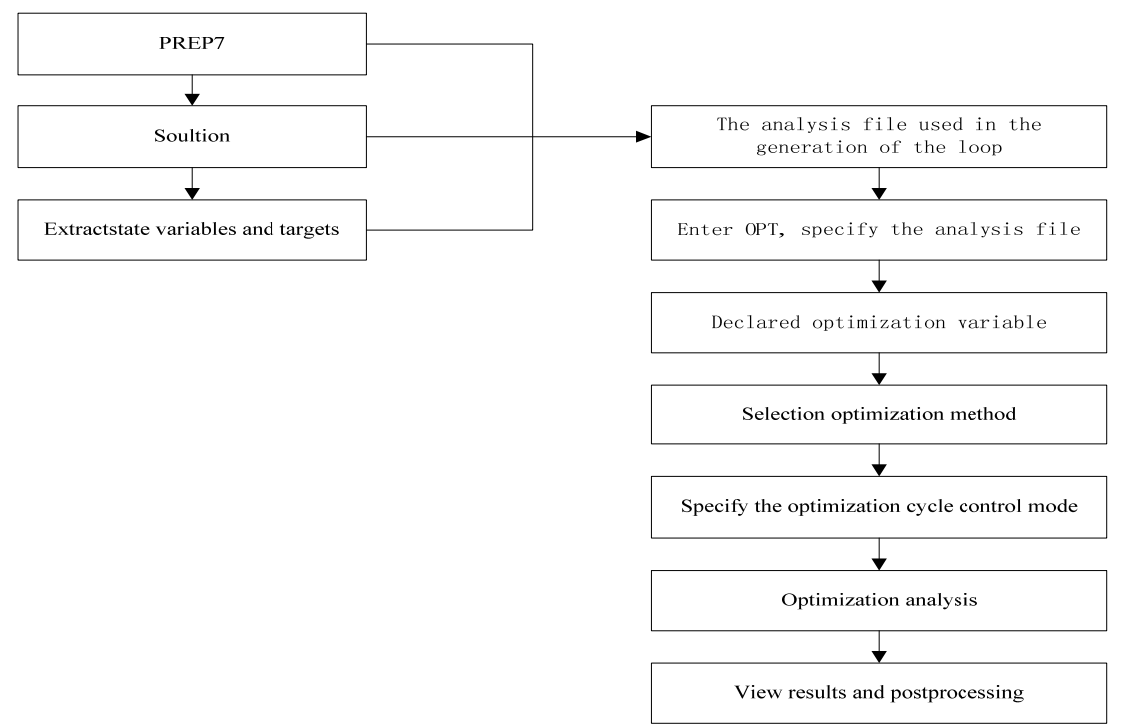

Figure 1 ANSYS optimization process

In Eq. 1: Q is a non binding objective function

$$
Q_{p}(x, q)=\sum_{i=1}^{n} P_{x}\left(x_{i}\right)+q\left[\sum_{i=1}^{m_{1}} P_{g}\left(g_{i}\right)+\sum_{i=1}^{m_{2}} P_{h}\left(h_{i}\right)+\sum_{i=1}^{m_{3}} P_{w}\left(w_{i}\right)\right]
$$

For the optimization of the i step, the optimal search direction $d^{[\sigma]}$ is introduced, then the next step is to design variable value:

$$
x^{(j+1)}=x^{(j)}+S_{j} d^{(j)}
$$

The $S_{j}$ is a linear search parameter, which is the most small step value on the search direction $\mathrm{d}^{(\mathrm{j})}$. It uses the gold partition ratio and the local square fitting technique to determine. Its range can be determined by Eq. 4 :

$$
0 \leq S_{j} \leq S_{\max } / 100 \times S_{j}
$$

The $S_{\max }$ is the maximum available step value, and the $S_{j}$ is the step value we chose, $S_{\text {max }} \in(0,100]$.

According to the Eq. 5, the search direction can be divided into two parts (Eq. 6 and Eq. 7 ) and the two parts can be determined by Eq. 8:

$$
\begin{gathered}
d^{j}=d_{f}^{j}+d_{p}^{j} \\
d_{f}^{j}=-\nabla Q_{f}\left(X^{j}\right)+r_{j-1} d_{f}^{j-1} \\
d_{p}^{j}=-q \nabla Q_{f}\left(X^{j}\right)+r_{j-1} d_{p}^{j-1} \\
r_{j-1}=\frac{\left[\nabla Q\left(x^{j}\right)-\nabla Q\left(X^{j-1}\right)\right]^{T} \nabla Q(X)}{\left|\nabla Q\left(X^{j-1}\right)\right|^{2}}
\end{gathered}
$$

Form Eq. 9, we can get gradient vector $\nabla Q\left(X^{j}\right)$ : 


$$
\nabla Q\left(X_{j}\right) \approx \frac{Q\left(x^{(j)}+\Delta X_{i e}\right)-Q\left(X^{(J)}\right)}{\Delta X_{i}}
$$

In Eq. 9: e is unit volume. It is 1 at the place of $\mathrm{i}$ and 0 at other place. $\Delta X_{i}=\frac{\Delta D}{100}\left(X^{n}-X^{1}\right)$ is the differential step size.

\section{Case analysis}

(1) The basic situation of the case

As shown in Fig. 2, the upper plane of the grid structure is composed of 36 nodes spacing $1 \mathrm{~m}$ and the lower plane is composed of 25 nodes spacing $1 \mathrm{~m}$, the distance between the upper and lower plane is $0.7 \mathrm{~m}$. The elastic modulus of the grid truss is $2.07 \mathrm{e} 11 \mathrm{~Pa}$ and the Poisson's ratio is 0.3 . In the work, the edge nodes of the grid are fixed, and each node of the lower plane is subjected to the vertical load $10 \mathrm{KN}$. Under the working condition, the maximum vertical displacement of the lower plane nodes is not more than $1 \mathrm{~cm}$, and the section stress of each member should be less than the allowable stress 210MPa. In order to make the structure of the whole grid material consumption to the least, the cross section of the rod of the the grid structure shoule be optimized.

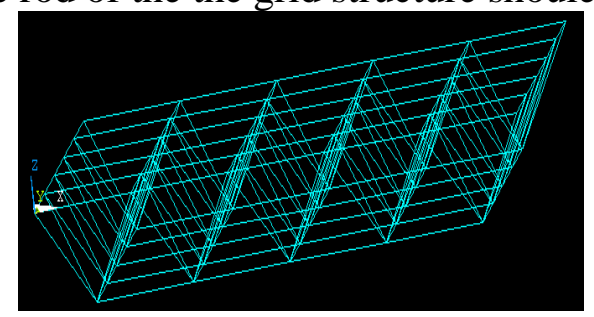

Figure 2 Space truss model

(2) The establishment of mathematical model and ANSYS optimization solution

The dimension parameters of the cross section of the member are designed according to the nature of the design variables in the actual engineering. Unified unit: $\mathrm{mm}$ and $\mathrm{N}$.

The establishment of mathematical optimization model:

Design variables

$$
\begin{aligned}
& \min T V O T=\sum_{i} A R E A \times l_{i} \\
& \text { s. t. } \sigma_{\max } \leq 210 \mathrm{MPa} \\
& \delta_{\max } \leq 10 \mathrm{~mm}
\end{aligned}
$$

State variables

ANSYS optimal operation steps:

Defining parameters. AREA=120

Defining element type. The plane beam element spar 3.

Defined real constant. Cross section area is AREA

Define material properties. E=2.07e11pa, Poisson's ratio is 0.3

Finite element model. Finite element model is generated by defining the nodes and elements.

Impose support constraint. The upper plane is surrounded by a fixed constraint.

Applied load. Under the plane each node is subject to the concentration of $10 \mathrm{KN}$

Solving.

Generate optimized analysis file

Optimization analysis file into the optimization design module. Declare design variables, state variables, objective functions, and specify the range of variables.

Select the optimization tools and methods for optimization.

View optimization results 


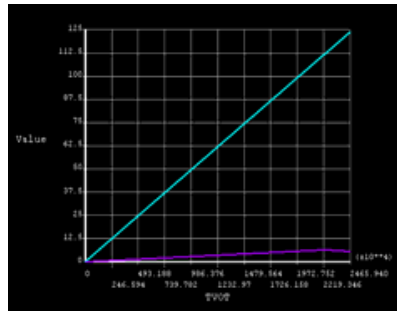

Figure 3 structural volume and section, displacement curves

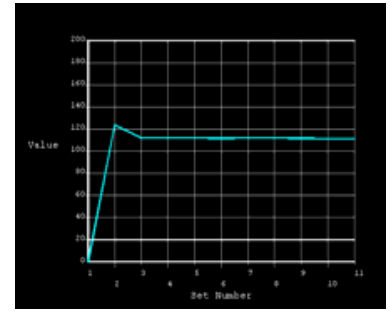

Figure 4 Structural change curves

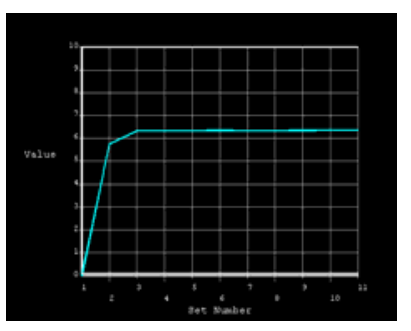

Figure 5 Structural displacment curves

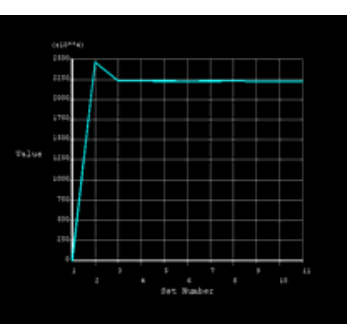

Figure 6 Structural volume curves

\begin{tabular}{|c|c|c|c|c|c|}
\hline $\begin{array}{l}\text { DMAX } \\
\text { SMAX } \\
\text { AREA } \\
\text { TUOT }\end{array}$ & $\begin{array}{l}\langle\mathbf{S U}\rangle \\
\langle\mathbf{S U}\rangle \\
\langle\mathrm{DU}\rangle \\
\langle\mathrm{OB} \boldsymbol{\rangle}\rangle\end{array}$ & 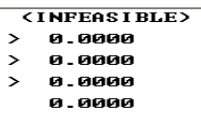 & $\begin{array}{c}\text { 〈I NFEAS I BLE〉 } \\
5.7287 \\
>189.45 \\
123.61 \\
0.24659 E+08\end{array}$ & $\begin{array}{l}\text { 〈FEAS I BLE) } \\
6.3191 \\
208-98 \\
112.06 \\
0.22356 E+08\end{array}$ & $\begin{array}{l}\text { 〈FEASIBLE> } \\
6.3291 \\
209.31 \\
111.88 \\
0.22320 E+08\end{array}$ \\
\hline & & $\begin{array}{c}\text { SET } 5 \\
\langle\text { FEAS I BLE }>\end{array}$ & $\begin{array}{c}\text { SET } 6 \\
\langle\text { FEAS I BLE > }\end{array}$ & $\begin{array}{c}\text { SET } \vec{?} \\
\text { 〈FEAS I BLE> }\end{array}$ & $\begin{array}{c}\text { SET } \\
\langle\text { FEAS I BLE }\rangle\end{array}$ \\
\hline $\begin{array}{l}\text { DMAX } \\
\text { smax }\end{array}$ & $\begin{array}{l}\langle s u\rangle \\
\langle s u\rangle\end{array}$ & $\begin{array}{l}6.3352 \\
209.51\end{array}$ & $\begin{array}{l}6.3414 \\
209.72\end{array}$ & $\begin{array}{l}6.3355 \\
209.52\end{array}$ & $\begin{array}{r}6.3295 \\
209.32\end{array}$ \\
\hline $\begin{array}{l}\text { ARER } \\
\text { TUOT }\end{array}$ & $\langle D U\rangle$ & $\begin{array}{l}111-79 \\
1109\end{array}$ & $111-66$ & $111-72$ & $111-87$ \\
\hline & & $\begin{array}{l}\text { D. } \\
\text { SET } 9 \\
\text { 〈FEAS I BLE> }\end{array}$ & $\begin{array}{l}\text { SET 10 } \\
\text { SET I BLE〉 } \\
\text { 〈FEAS I }\end{array}$ & $\begin{array}{l}\text { *SET 11* } \\
\text { *FEAS I BLE> }\end{array}$ & \\
\hline & $\langle s u\rangle$ & 6.3395 & 6.3457 & $6-3481$ & \\
\hline $\operatorname{smax}$ & $\langle s u\rangle$ & 209.65 & 209.86 & $209-94$ & \\
\hline AREA & $\langle D U\rangle$ & $111-70$ & 111 . 59 & 111 -55 & \\
\hline тUот & $\langle O B J\rangle$ & D $-22284 E+68$ & $0.22262 E+08$ & $0.22254 E+08$ & \\
\hline
\end{tabular}

Figure 7 Optimization results

Therefore, the final optimization results for the cross-sectional area is $112 \mathrm{~mm}^{2}$ and the final volume $2.24 \mathrm{E} 07 \mathrm{~mm}^{3}$. At this time, the maximum stress and the maximum displacement of the structure both meet the requirements of the condition. The volume reduces by $6.7 \%$, so the cost will be reduced accordingly.

\section{Conclusion}

The paper chose the grid structure with extensive engineering significance as the research object and use ANSYS to optimize the parameters of the target. Through the theoretical analysis and the example of the ANSYS optimization, the feasibility of the optimization analysis of ANSYS software is described. Through the iterative calculation, the results which meeting the repuiremtnes of the conditions are the optimal choice comparing to the previous. Therefore, the optimization theory and the corresponding optimization software have certain reference value in practical engineering application.

\section{Acknowledgement}

This research was financially supported by the National Education Foundation (BIA110076) and Science and Technology Foundation of Wuhu (2012cxy08).

\section{References}

[1] X.W. Wang, Z.J. Yang, L. Duan. Discussion on some problems of ANSYS optimization design, J. Journal of plastic engineering. 14(6). 181-184(2007)

[2] X.L. Bai. Structural optimization design, Zheng Zhou, 2008

[3] Tanskanen P. The evolutionary structural optimization: theoretical aspects[J] Computer Methods in Applied Mechanics and Engineering. 191(47-48): 5485-5498(2002)

[4] X. Zhao, Y.L. Tai. Structure design optimization of ANSYS. Journal of Liaoning Technical University J. 25. 144-146(2006) 